\title{
Posterior scleritis as an intraocular tumour
} Paul T Finger, Henry D Perry, Samuel Packer, Richard A Erdey, Geoffrey D Weisman,
Patrick A Sibony

\begin{abstract}
Posterior scleritis rarely presents as a large 'intraocular tumour' and continues to be a challenge in diagnosis and management. We present the case of a 66-year-old male with no known rheumatic disease, pain, proptosis, or diplopia, but with progressively decreasing visual acuity and a large, discrete 'subretinal tumour', $15 \times 17 \mathrm{~mm}$ basal diameter and $8.3 \mathrm{~mm}$ apical height. Histologically the tumour was shown to be grossly thickened and necrotic sclera.
\end{abstract}

Posterior scleritis is difficult to diagnose because of its tendency to present with a variety of symptoms. ${ }^{1}$ These symptoms include pain, refractive changes, decreased visual acuity, diplopia, and proptosis. ${ }^{2-6}$ Though a history of collagen vascular disease, anterior scleritis, female sex, or serous retinal detachment can aid in the diagnosis, none is universally characteristic. ${ }^{6}$ When posterior scleritis presents as an 'intraocular tumour', A- and B-scan ultrasonography showing relatively high internal reflectivity, thickened sclera, and retrobulbar oedema may be the most useful ancillary tests. ${ }^{45}$

\section{Case report}

A 66-year-old while male presented with a fiveyear history of 'red eyes' and a two-year history of decreased vision OS. He had a history of a transient episode of polyarthritis and unexplained jaundice five years before presentation, blepharitis in both eyes, and glaucoma in the left eye.

Ophthalmic examination revealed a visual

Ophthalmic Oncology Service, Department of Ophthalmology, North Shore University Hospital - Cornell University Medical College, Manhasset, New York; Department of Ophthalmology, State University of New York at Stony Brook, Stony Brook, New York P T Finger H D Perry

S Packer

R A Erdey

G D Weisman

P A Sibony

Correspondence to: Paul T Finger, MD, Ophthalmic Oncology Service, North Shore University Hospital Cornell University Medical College, 300 Community Drive, Manhasset, New York 11030, USA.

Accepted for publication 11 October 1989

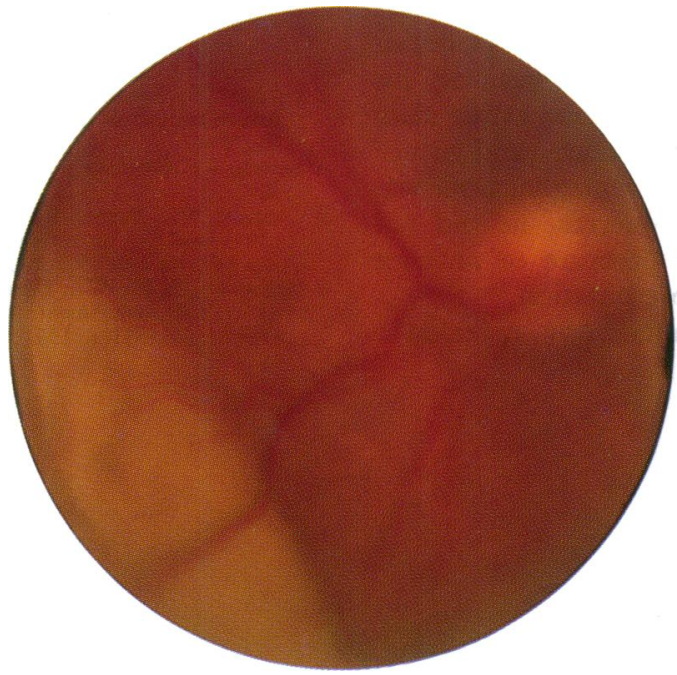

Figure 1: Through a hazy vitreous an orange-white superonasal subretinal mass extends to within $3 \mathrm{~mm}$ of the optic nerve.

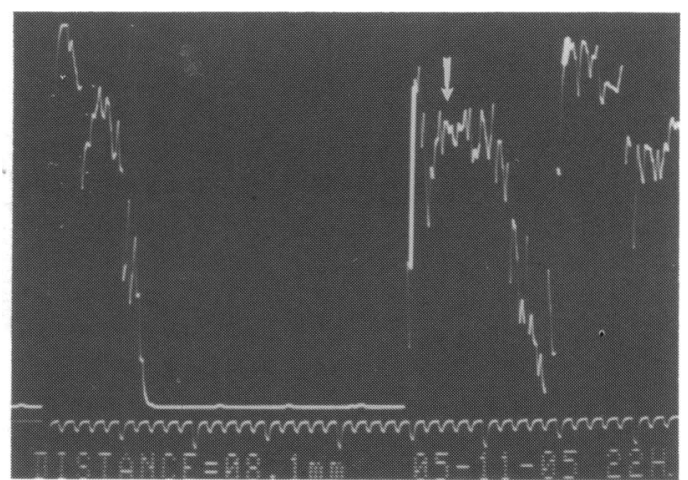

Figure 2: A-scan echogram showing an apical tumour height of $8.1 \mathrm{~mm}$, and relatively high intratumor reflectivity (arrow).

acuity of 20/25 OD and 20/200 OS, an afferent pupillary defect OS, full ocular motility, and applanation tensions of $14 \mathrm{OD}$ and 12 OS. Slitlamp examination showed a mild seborrhoeic blepharitis, conjunctival injection, mild scleral thinning $4 \mathrm{~mm}$ posterior to the superonasal limbus OS, and posterior subcapsular cataracts OU. Indirect ophthalmoscopy revealed vitreous cells, a large, non-rhegmatogenous retinal detachment in the superonasal quadrant, and an underlying orange-white tumour $15 \times 17 \mathrm{~mm}$ in basal diameter and extending to within $3 \mathrm{~mm}$ of the optic nerve OS (Fig 1).

A-scan ultrasonography revealed relatively high intratumour reflectivity and a tumour height of $8 \cdot 1 \mathrm{~mm}$ (Fig 2). B-scan ultrasonography showed a subretinal tumour with a multinodular surface, with a non-homogeneous intratumour pattern, surrounded by a retrobulbar echoluscent zone (Fig 3). On fluorescein angiography blotchy hyperfluorescence over the tumour and cystoid macular oedema were noted OS.

Our patient was advised of the possibility that this tumour could be a choroidal melanoma with some atypical characteristics. A metastatic survey was negative and the options of observation, radiation, and enucleation were discussed.

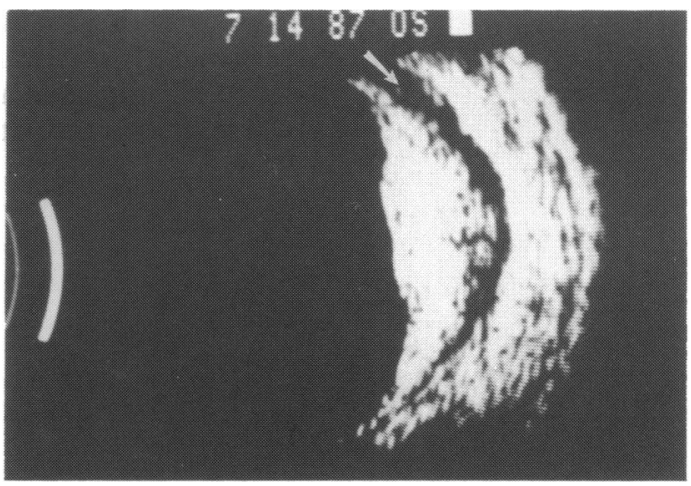

Figure 3: B-scan echogram showing the intraocular tumour with a multinodular surface, internal reflectivity, and retrobulbar echolucency (arrow). 


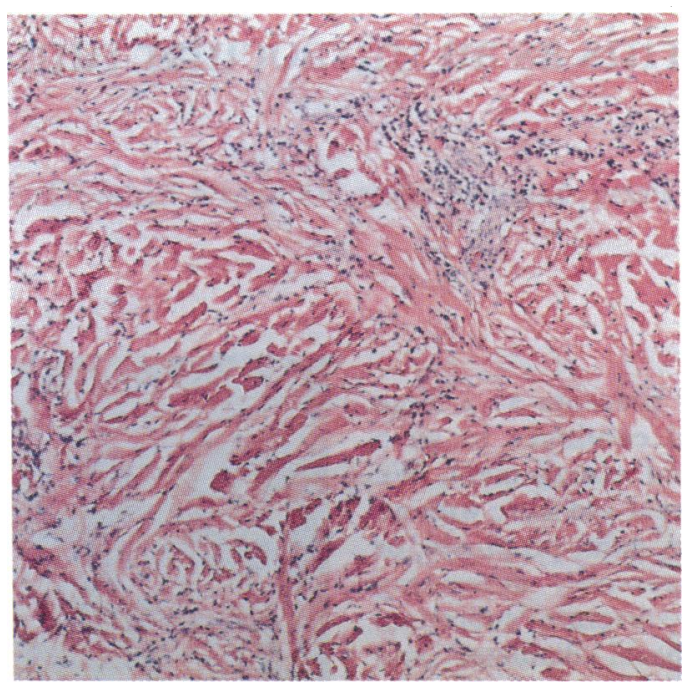

Figure 4: Histological section revealed diffuse oedema and inflammation separating scleral collagen fibres.

(Haematoxylin and eosin, $\times 35$ )

He chose to be enucleated and have $600 \mathrm{cGy}$ of preoperative external beam radiotherapy.

\section{PATHOLOGICAL FINDINGS}

While scleral thinning was noted superiorly from the 8 o'clock to the 12 o'clock positions running between $4 \mathrm{~mm}$ and $8 \mathrm{~mm}$ posterior to the limbus, the sclera showed marked thickening in an area measuring $15 \times 17 \mathrm{~mm}$ in basal diameter and reaching $8.3 \mathrm{~mm}$ in maximal thickness. By light microscopy the intraocular mass was noted to arise from the sclera, which was thickened and infiltrated with inflammatory cells. Diffuse oedema separated scleral collagen fibres (Fig 4), and there were nodular areas of necrosis with a granulomatous reaction next to necrotic sclera (Fig 5). A large serous detachment of the retina was also noted in association with multifocal retinal pigment epithelial detachments.

\section{Discussion}

It is noteworthy that our patient did not have ocular pain. His injected conjunctiva was attributable to blepharitis, and his decreased visual acuity was secondary to cystoid macular oedema, an atypical finding for posterior scleritis. ${ }^{4} \mathrm{He}$ lacked proptosis, because his 'tumour' had grown into the globe rather than extending into the muscles, cone, and orbit. ${ }^{4}$ Though his history of transient glaucoma was consistent with posterior scleritis, he did not have a raised intraocular pressure or anterior segment inflammation at presentation. ${ }^{46}$

While indirect ophthalmoscopy revealed a

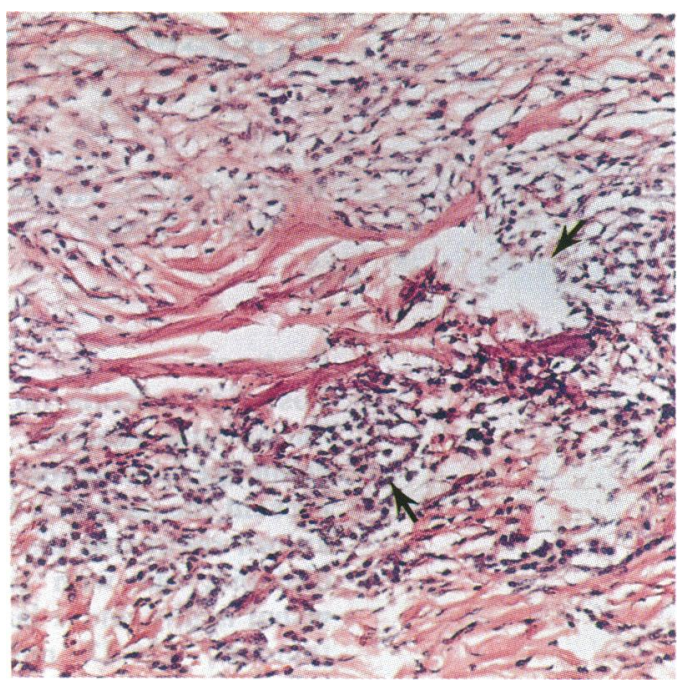

Figure 5: Histological section demonstrating areas of scleral necrosis and granulomatous infiltrates (arrows).

(Haematoxylin and eosin, $\times 35$ )

large superonasal orange elevation of the choroid, choroidal folds and retinal striae secondary to diffuse posterior scleral inflammation were notably absent.

Ultrasonography was most consistent with the diagnosis of posterior scleritis. Though the A-scan showed an unusually large intraocular tumour, intratumour reflectivity was very high. This was probably due to the many echodense scleral fibres separated by regions of inflammation and necrosis seen on histological examination (Fig 5). Our B-scan findings were similar to those of Cappaert et al, ${ }^{5}$ showing thickened sclera and retrobulbar oedema (Fig 3).

In one of the earliest documented cases of posterior scleritis a 49-year-old male presented with a painful red eye, light perception only retinal detachment, and secondary glaucoma, and was diagnosed by enucleation. ${ }^{6}$ Our remarkably similar case, occurring over 50 years later, illustrates that, in spite of the modern diagnostic aids of fluorescein angiography and ultrasonography, posterior scleritis as an intraocular tumour' remains a diagnostic challenge.

1 Singh G, Guthoff R, Foster CS. Observations on the long-term follow-up of posterior scleritis. Am F Ophthalmol 1986; 101 570-5.

2 Benson WE, Shields JA, Tasman W, Crandall AS. Posterior scleritis: a cause of diagnostic confusion. Arch Ophthalmol 979; 97 : $1482-6$.

3 Feldon SE, Sigelman J, Albert DM, Smith TR. Clinical manifestations of brawny scleritis. Am $\mathcal{F}$ Ophthalmol 1978; 85: 781-7.

4 Benson WE. Posterior scleritis. Surv Ophthalmol 1988; 32: 297 316.

5 Cappaert WE, Purnell EW, Frank KE. Use of B-sector scan ultrasound in the diagnosis of benign choroidal folds. Am $\mathcal{F}$ Ophthalmol 1977; 84: 375-9.

6 Holloway TB, Fry WE. Unsuspected brawny scleritis in a case of retinal detachment with secondary glaucoma. Arch Ophthalmol 1931; 6: 136 . 